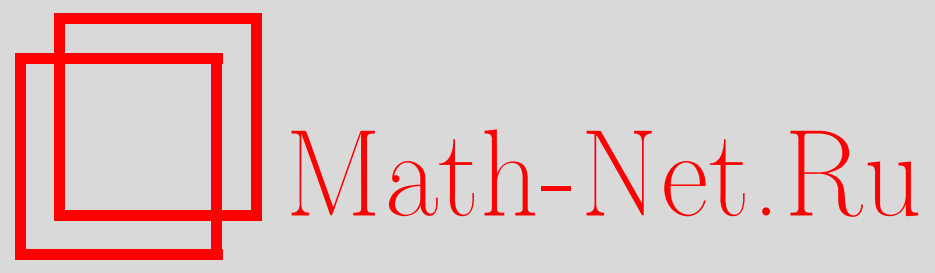

А. М. Шойтов, Предельные распределения числа наборов $H$-эквивалентных отрезков в равновероятной полиномиальной схеме серий, Дискрет. матем., 2002, том 14, выпуск 1, 82-98

DOI: https://doi.org/10.4213/dm233

Использование Общероссийского математического портала Math-Net.Ru подразумевает, что вы прочитали и согласны с пользовательским соглашением http: //www.mathnet.ru/rus/agreement

Параметры загрузки :

IP : 18.234 .156 .22

26 апреля 2023 г., 15:38:54

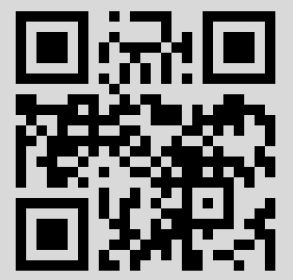




\title{
Предельные распределения числа наборов $H$-эквивалентных отрезков в равновероятной полиномиальной схеме серий
}

\author{
(C) 2002 г. $\quad$ А. М. Шойтов
}

\begin{abstract}
В работе изучаются случайные величины, характеризующие связь наборов отрезков равновероятной полиномиальной схемы отношением $H$-эквивалентности. Получена верхняя оценка расстояния по вариации между распределением случайной величины $\xi_{k}(H)$, равной числу наборов $H$-эквивалентных отрезков, и распределением Пуассона. Найдены достаточные условия сходимости функции распределения числа $H$-эквивалентных отрезков в схеме серий равновероятных полиномиальных испытаний к нормальному распределению, распределению Пуассона и к сложному распределению Пуассона.
\end{abstract}

Пусть $X=X_{1}, X_{2}, \ldots$ - последовательность случайных величин (с.в.), принимающих значения из множества $A=\{1,2, \ldots, v\}, v>1$, полученная по равновероятной полиномиальной схеме.

Для натурального $n$ будем говорить (см. [8]), что два вектора $\bar{a}=\left(a_{1}, a_{2}, \ldots, a_{n}\right)$ и $\bar{b}=\left(b_{1}, b_{2}, \ldots, b_{n}\right)$ из $A^{n} H$-эквивалентны относительно некоторой подгруппы $H$ симметрической группы подстановок $S(A)$, если существует такая подстановка $h \in H$, что $h\left(a_{j}\right)=b_{j}$ для всех $j=1, \ldots, n$. В этом случае будем писать $\bar{a} H \bar{b}$.

Для натурального $k \geqslant 2$ и произвольных натуральных $1 \leqslant i_{1}<i_{2}<\ldots<i_{k} \leqslant N$ определим с.в. $\eta\left(i_{1}, \ldots, i_{k} ; H, n\right)$, полагая

$$
\eta\left(i_{1}, \ldots, i_{k} ; H, n\right)=I\left(\forall r, s=1, \ldots, k:\left(X_{i_{r}}, \ldots, X_{i_{r}+n-1}\right) H\left(X_{i_{s}}, \ldots, X_{i_{s}+n-1}\right)\right),
$$

где $I(C)$-- индикатор события $C$. Рассмотрим с.в.

$$
\xi_{k}=\xi_{k}(N)=\xi(H, k, N, n)=\sum_{1 \leqslant i_{1}<\ldots<i_{k} \leqslant N} \eta\left(i_{1}, \ldots, i_{k} ; H, n\right),
$$

равные числу наборов из $k H$-эквивалентных отрезков длины $n$ в последовательности $X$ длины $N+n-1$.

Предельное распределение с.в. $\xi(H, k, N, n)$ при $H=\{e\}$ исследовалось в работах А. М. Зубкова и В. Г. Михайлова (см., например, $[3,6])$. В работах С. М. Буравлева (см. $[1,2])$ рассматривались случаи $H=S(A)$ и $H$ - латинский прямоугольник. 
В данной работе исследуется случай произвольной группы подстановок $H$. Близкой к описанной задаче является задача о числе $H$-эквивалентных отрезков в двух различных последовательностях, рассмотренная в [7].

Введем обозначения. Пусть $\nu_{m}(B)-$ множество всех бесповторных $m$-выборок элементов множества $B$. Для всех $m=1, \ldots, v$ обозначим через $\operatorname{Orb}(m)$ множество классов $H$-эквивалентных элементов $\nu_{m}(A)$.

Пусть $\Pi_{\lambda}(x)$ - функция распределения закона Пуассона с параметром $\lambda, \Phi(x)-$ функция распределения стандартного нормального закона, и пусть $F_{\xi_{k}}(x)$ - функция распределения случайной величины $\xi_{k}$. Кроме того, обозначим через $d(F, G)$ расстояние по вариации между функциями распределения $F$ и $G$ (см. [9]).

Положим

$$
\begin{aligned}
& \delta=\delta(n)=\frac{1}{v^{k n}} \sum_{m=1}^{v} \sigma(n, m) \sum_{f \in \operatorname{Orb}(m)}|f|^{k}, \\
& \Delta=\Delta(n)=\frac{|H|}{v^{n}} \\
& \lambda_{1}=\lambda_{1}(H, n, N)=\left(\begin{array}{c}
N \\
k
\end{array}\right) \delta \\
& \lambda_{2}=\lambda_{2}(H, n, N)=\left(\begin{array}{c}
N \\
k
\end{array}\right) \Delta^{k-1}
\end{aligned}
$$

где $\sigma(n, m)$ - числа Стирлинга второго рода.

Теорема 1. Ecлu $n / v \geqslant \ln v+1$, mo

$$
\begin{aligned}
d\left(F_{\xi_{k}}, \Pi_{\lambda_{1}}\right) \leqslant\left(\Psi+\lambda_{1}\left(3 \psi+(2 n)^{k}(N \Delta)^{k-1}\right)+12 \lambda_{2} / v\right) & \\
& \times\left(\left(\frac{1-e^{\lambda_{1}}}{\lambda_{1}}\right)+\min \left\{1,\left(\min \left\{\lambda_{1},\left|\lambda_{1}-\psi\right|\right\}\right)^{-2}\right\} \psi\right),
\end{aligned}
$$

гдe

$$
\begin{aligned}
& \psi=(2 n)^{k-1} N \Delta \exp (N \Delta / n) \\
& \Psi=(4 n)^{2 k-1} N \Delta \exp (N \Delta)
\end{aligned}
$$

при этом $\lambda_{1}<\lambda_{2}$

Следствие 1. Пустъ $N, n, v \rightarrow \infty \max$, ито $n / v-\ln v \rightarrow \infty$, moгда $\lambda_{1}=\lambda_{2}(1+o(1))$. Если, кроме того, въполнено условие

$$
\varphi=(4 n)^{2 k-1} N \Delta \rightarrow 0
$$

mo

$$
d\left(F\left(\xi_{k}\right), \Pi\left(\lambda_{1}\right)\right)=O\left(\max \left(\varphi, \lambda_{2} / v\right)\right)
$$

если $0<\lambda_{0}=\varliminf_{N \rightarrow \infty} \lambda_{1}$, то для въполнения (3) достаточно, чтобъ

$$
\phi=\lambda_{2} k(4 n)^{2 k-1} \Delta^{1 / k} \rightarrow 0
$$


Теорема 2. Пусть $N, n, v \rightarrow \infty$ max, ито $n / v-\ln v \rightarrow \infty$, существует предел $\lambda_{0}=\lim _{N \rightarrow \infty} \lambda_{1}, 0<\lambda_{0} \leqslant \infty, u$ выполнено условие (3) следствия 1 .

Тогда при $\lambda_{0}<\infty$

$$
\mathbf{P}\left(\xi_{k}=r\right) \rightarrow \frac{\lambda_{0}^{r}}{r !} e^{-\lambda_{0}}, \quad r=0,1, \ldots
$$

npu $\lambda_{0}=\infty, \lambda_{2} / v \rightarrow 0$

$$
\mathbf{P}\left(\left(\xi_{k}-\lambda_{1}\right) / \sqrt{\lambda_{1}}<x\right) \rightarrow \Phi(x),
$$

если $\lambda_{2} / v \rightarrow 0$, то условие (3) въполнено при $k=o(\sqrt{n \ln v / \ln n})$, в частности, при фиксированном $k$.

Замечание 1. Для ряда случаев выбора множества $H$, например, когда $H=\{e\}$ (см. [6]) или $H-$ латинский прямоугольник (см. [2]), условие типа $n / v-\ln v \rightarrow \infty$ теоремы 2 является необходимым для сходимости функции распределения случайной величины $\xi_{k}$ к функции распределения Пуассона. Однако, видимо, для больших по мощности групп $H$ условие подобного рода не является необходимым.

Замечание 2. Условия сходимости распределения случайной величины $\xi_{k}$ к сложному распределению Пуассона (случай фиксированного $v$ ) установлены в теореме 3 (см. ниже).

Доказательство теоремъ 1. Далее предполагаем, что $n / v \geqslant \ln v+1$. С помощью несложных комбинаторных рассуждений нетрудно убедиться, что в случае, когда $\left|i_{s}-i_{r}\right| \geqslant n$ для всех $s \neq r$, выполнено равенство $\mathrm{E} \eta_{k}\left(i_{1}, \ldots, i_{k}\right)=\delta$. Кроме того, для всех $m=1, \ldots, v$ имеет место неравенство $|f| \leqslant|H|$ при $f \in \operatorname{Orb}(m)$ и $|f|=|H|$ при $f \in \operatorname{Orb}(v)$. Отсюда

$$
\begin{aligned}
\delta & =\frac{1}{v^{k n}} \sum_{m=1}^{v} \sigma(n, m) \sum_{f \in \operatorname{Orb}(m)}|f|^{k} \leqslant \frac{|H|^{k-1}}{v^{k n}} \sum_{m=1}^{v} \sigma(n, m) \sum_{f \in \operatorname{Orb}(m)}|f| \\
& =\frac{|H|^{k-1}}{v^{k n}} \sum_{m=1}^{v} \sigma(n, m)(v)_{m}<\left(\frac{|H|}{v^{n}}\right)^{k-1}=\Delta^{k-1}, \\
\delta & =\frac{1}{v^{k n}} \sum_{m=1}^{v} \sigma(n, m) \sum_{f \in \operatorname{Orb}(m)}|f|^{k}>\frac{1}{v^{k n}} \sigma(n, v) \sum_{f \in \operatorname{Orb}(v)}|f|^{k} \\
& =\left(\frac{|H|}{v^{n}}\right)^{k-1} \frac{\sigma(n, v) v !}{v^{n}}=\Delta^{k-1} \frac{\sigma(n, v) v !}{v^{n}} .
\end{aligned}
$$

Таким образом, $\lambda_{1}<\lambda_{2}$, кроме того, при $n / v-\ln (v) \rightarrow \infty$ согласно [5]

$$
\sigma(n, v)=\frac{v^{n}}{v !}(1+o(1))
$$

и, следовательно, $\lambda_{1}=\lambda_{2}(1+o(1))$.

Далее при доказательстве мы будем использовать метод Чена-Стейна оценки точности пуассоновской аппроксимации (см. [10]) и придерживаться в ряде случаев схем рассуждений, изложенных в [1] и [6]. Для краткости случайную величину $\eta\left(i_{1}, \ldots, i_{k} ; n, N\right)$ будем обозначать через $\eta(\bar{i})$, где $\bar{i}=\left(i_{1}, \ldots, i_{k}\right)$. 
Пусть $J=\left\{\bar{i}: \bar{i}=\left(i_{1}, \ldots, i_{k}\right), 1 \leqslant i_{1}<\ldots<i_{k} \leqslant N\right\}, \lambda=\mathbf{E} \xi_{k}(n, N)$, и для любого $\bar{i} \in J$ пусть $J(\bar{i})$ - множество таких наборов $\bar{j} \in J, \bar{j} \neq \bar{i}$, что хотя бы один из отрезков $\left[i_{1}, i_{1}+n-1\right], \ldots,\left[i_{k}, i_{k}+n-1\right]$ пересекается хотя бы с одним из отрезков $\left[j_{1}, j_{1}+n-1\right], \ldots,\left[j_{k}, j_{k}+n-1\right]$. Тогда согласно теореме $10 . \mathrm{A} \mathrm{работы} \mathrm{[10]}$

$$
d\left(F_{\xi_{k}}, \Pi_{\lambda}\right) \leqslant \frac{1-e^{-\lambda}}{\lambda} \Lambda
$$

где

$$
\Lambda=\sum_{\bar{i} \in J} \mathbf{E} \eta(\bar{i})\left(\mathbf{E} \eta(\bar{i})+\sum_{\bar{j} \in J(\bar{i})} \mathbf{E} \eta(\bar{j})\right)+\sum_{\bar{i} \in J} \sum_{\bar{j} \in J(\bar{i})} \mathbf{E}(\eta(\bar{j}) \eta(\bar{i})) .
$$

Для любого $\bar{i} \in J$ определим события

$$
B(\bar{i})=\{\eta(\bar{i})=1\}
$$

и множества

$$
U(\bar{i})=\bigcup_{t=1}^{k}\left[i_{t}, i_{t}+n-1\right]
$$

кроме того, для любого $\bar{j} \in J, \bar{j} \neq \bar{i}$, пусть

$$
U(\bar{i}, \bar{j})=U(\bar{i}) \cup U(\bar{j}) .
$$

Обозначим через $A(\bar{i}, \bar{j})$ и $A(\bar{i})$ число связных компонент (отрезков) множеств $U(\bar{i}, \bar{j})$ и $U(\bar{i})$ соответственно. Обозначим $J(\bar{i}, a)$ множество $\bar{j} \in J, \bar{j} \neq \bar{i}$, таких, что существует ровно $а$ компонент множества $U(\bar{i}, \bar{j})$, не пересекающихся с $U(\bar{i})$. Обозначим $J(a)$ множество $\bar{i} \in J$ таких, что множество $U(\bar{i})$ состоит ровно из $a$ связных компонент. Очевидно,

$$
J=\bigcup_{a=1}^{k} J(a)
$$

Лемма 1. Пусть $\bar{i} \in J(a)$. Если $1 \leqslant a<k$, mo

$$
\mathbf{P}\{B(\bar{i})\} \leqslant \Delta^{a},
$$

если $a=k$, mo

$$
\mathbf{P}\{B(\bar{i})\}=\delta .
$$

$\Pi y c m$ ๖ $(\bar{i}, \bar{j}) \in J(\bar{i}, a)$. Ecлu $0 \leqslant a<k$, mo

$$
\mathbf{P}\{B(\bar{i}) \mid B(\bar{j})\} \leqslant \Delta^{a},
$$

если $a=k$, mo

$$
\mathbf{P}\{B(\bar{i}) \mid B(\bar{j})\}=\delta .
$$

Доказателъство. Утверждения (6) и (8) доказаны выше. Для доказательства (7) заметим, что, так как $a<k$, существует отрезок $\left[i_{m}, i_{m}+n-1\right], m=1, \ldots, k$, пересекающийся либо с другим из отрезков $\left\{\left[i_{1}, i_{1}+n-1\right], \ldots,\left[i_{k}, i_{k}+n-1\right]\right\}$, либо 
с множеством $U(\bar{j})$. Пусть $\left[i_{m_{1}}, i_{m_{1}}+n-1\right], \ldots,\left[i_{m_{a}}, i_{m_{a}}+n-1\right]-$ отрезки, содержащиеся в различных компонентах связности $U(\bar{i})$, не пересекающихся с $U(\bar{j})$. Тогда

$$
\begin{aligned}
\mathbf{P}\{B(\bar{i}) \mid B(\bar{j})\} \leqslant & \mathbf{P}\left\{Y\left(i_{m}\right) H Y\left(i_{m_{s}}\right), s=1, \ldots, a \mid B(\bar{j})\right\} \\
= & \sum_{\substack{\left(y_{1}, \ldots, y_{n}\right) \in A^{n}\\
}} \mathbf{P}\left\{Y\left(j_{m}\right)=\left(y_{1}, \ldots, y_{n}\right) \mid B(\bar{j})\right\} \\
= & \sum_{\substack{\left(y_{1} \ldots, y_{n}\right) \in A^{n}\\
}} \mathbf{P}\left\{Y\left(j_{m}\right) H Y\left(j_{m_{s}}\right), s=1, \ldots, a \mid B(\bar{j}), Y\left(j_{m}\right)=\left(y_{1}, \ldots, y_{n}\right)\right\} \\
& \mathbf{P}\left\{Y\left(j_{m}\right) H Y\left(j_{m_{s}}\right), s=1, \ldots, a \mid Y\left(j_{m}\right)=\left(y_{1}, \ldots, y_{n}\right)\right\} \leqslant \Delta^{a} .
\end{aligned}
$$

Доказательство (5) аналогично.

Применяя лемму 1 , получаем, что

$$
\sum_{\bar{i} \in J}(\mathbf{E} \eta(\bar{i}))^{2}=\sum_{a=1}^{k} \sum_{i \in J(a)}(\mathbf{E} \eta(\bar{i}))^{2} \leqslant \sum_{a=1}^{k-1}|J(a)| \Delta^{2 a}+\left(\begin{array}{c}
N \\
k
\end{array}\right) \delta^{2}
$$

В [6] показано, что

$$
|J(a)| \leqslant\left(\begin{array}{l}
N \\
a
\end{array}\right)\left(\begin{array}{c}
k-1 \\
a-1
\end{array}\right) n^{k-a}
$$

Поэтому

$$
\sum_{\bar{i} \in J}(\mathrm{E} \eta(\bar{i}))^{2} \leqslant \sum_{a=1}^{k-1}\left(\begin{array}{c}
N \\
a
\end{array}\right)\left(\begin{array}{c}
k-1 \\
a-1
\end{array}\right) n^{k-a} \Delta^{2 a}+\left(\begin{array}{l}
N \\
k
\end{array}\right) \delta^{2} .
$$

Оценим величину

$$
\sum_{a=1}^{k}\left(\begin{array}{l}
N \\
a
\end{array}\right)\left(\begin{array}{l}
k-1 \\
a-1
\end{array}\right) n^{k-a} D^{a}
$$

при $D<1$. Справедливы соотношения

$$
\begin{aligned}
\sum_{a=1}^{k-1}\left(\begin{array}{l}
N \\
a
\end{array}\right)\left(\begin{array}{c}
k-1 \\
a-1
\end{array}\right) n^{k-a} D^{a} & \leqslant(2 n)^{k-1} \sum_{a=1}^{k-1} \frac{N^{a}}{a !} n^{1-a} D^{a} \\
& \leqslant(2 n)^{k-1} N D \sum_{a=1}^{\infty} \frac{1}{(a-1) !}\left(\frac{N D}{n}\right)^{a-1} \\
& =(2 n)^{k-1} N D \exp \left(\frac{N D}{n}\right)
\end{aligned}
$$

Таким образом,

$$
\sum_{\bar{i} \in J}(\mathbf{E} \eta(\bar{i}))^{2} \leqslant(2 n)^{k-1} N \Delta^{2} \exp \left(\frac{N \Delta^{2}}{n}\right)+\lambda_{1} \delta \leqslant \psi \Delta+\lambda_{1} \delta .
$$

Аналогичными рассуждениями получаем неравенство $\left|\lambda_{1}-\lambda\right| \leqslant \psi$. 
Далее,

$$
\begin{aligned}
& \sum_{\bar{i} \in J} \mathbf{E} \eta(\bar{i}) \sum_{\bar{j} \in J(\bar{i})} \mathbf{E} \eta(\bar{j}) \leqslant 2 \sum_{a=1}^{k} \sum_{\bar{i} \in J(a)} \mathbf{E} \eta(\bar{i}) \sum_{b=1}^{k-1} \sum_{\bar{j} \in J(b)} \mathbf{E} \eta(\bar{j}) \\
& +\sum_{\bar{i} \in J(k)} \mathbf{E} \eta(\bar{i}) \sum_{c=0}^{k-1} \sum_{\bar{j} \in J(k) \cap J(\bar{i}, c)} \mathbf{E} \eta(\bar{j}) \\
& \leqslant 2\left(\sum_{a=1}^{k-1}|J(a)| \Delta^{a}+\left(\begin{array}{c}
N \\
k
\end{array}\right) \delta\right) \sum_{a=1}^{k-1}|J(a)| \Delta^{a} \\
& +\delta^{2}\left(\sum_{\bar{i} \in J(k)} \sum_{c=0}^{k-1} \sum_{\bar{j} \in J(k) \cap J(\bar{i}, c)} 1\right) \text {. }
\end{aligned}
$$

Замечая, что при $\bar{i} \in J(k)$ имеет место неравенство

$$
|J(k) \cap J(\bar{i}, c)| \leqslant\left(\begin{array}{l}
k \\
c
\end{array}\right)(2 n-1)^{k-c}\left(\begin{array}{l}
N \\
c
\end{array}\right),
$$

получаем, что

$$
\begin{aligned}
\sum_{i \in J} \operatorname{E} \eta(\bar{i}) \sum_{j \in J(\bar{i})} \mathbf{E}_{\eta}(\bar{j}) & \leqslant 2\left(\psi+\lambda_{1}\right) \psi+\delta^{2}\left(\begin{array}{l}
N \\
k
\end{array}\right) \sum_{a=0}^{k-1}\left(\begin{array}{l}
k \\
a
\end{array}\right)(2 n-1)^{k-a}\left(\begin{array}{l}
N \\
a
\end{array}\right) \\
& \leqslant 2\left(\psi+\lambda_{1}\right) \psi+\lambda_{1} \Delta^{k-1} N^{k-1} \sum_{a=0}^{k-1}\left(\begin{array}{l}
k \\
a
\end{array}\right)(2 n-1)^{k-a} \\
& \leqslant 2 \psi^{2}+\lambda_{1}\left(2 \psi+\left((2 n)^{k}-1\right)(N \Delta)^{k-1}\right) .
\end{aligned}
$$

Пусть $I=\{(\bar{i}, \bar{j}): \bar{i} \in J, \bar{j} \in J(\bar{i})\}$. Оценим

$$
\sum_{(\bar{i}, \bar{j}) \in I} \mathbf{E} \eta(\bar{j}) \eta(\bar{i})
$$

Введем следующие обонначения. Набор $(\bar{i}, \bar{j})$ назовем симметричным, если для всех $s=1, \ldots, k$ отрезки с началами $i_{s}$ и $j_{s}$ и только они принадлежат одной компоненте связности $U(\bar{i}, \bar{j}), i_{s}-j_{s}=i_{q}-j_{q}, s, q=1, \ldots, k$. Обозначим множество симметричных наборов через $I_{s}$, кроме того, пусть $I_{n s}=I \backslash I_{s}$ и

$$
\Psi^{\prime}=(4 n)^{2 k-1}(\exp (N \Delta)-1) \text {. }
$$

Лемма 2. В условиях теоремы 1

$$
\sum_{(\bar{i}, \bar{j}) \in I_{n \times}} \operatorname{E} \eta(\bar{j}) \eta(\bar{i}) \leqslant \lambda_{1} \psi+\Psi^{\prime}
$$

Доказательство. Разложим $I_{n s}$ на сумму непересекающихся множеств $L\left(a, a_{1}, a_{2}\right)$, положив

$$
L\left(a, a_{1}, a_{2}\right)=\left\{(\bar{i}, \bar{j}) \in I_{n s},\left(i_{1}, \ldots, i_{k}\right) \in J\left(a_{1}\right),\left(j_{1}, \ldots, j_{k}\right) \in J\left(a_{2}\right),\right.
$$
и имеется ровно а связных компонент множества $U(\bar{i}, \bar{j})\}$. 
Пользуясь леммой 1 , получаем, что для $(\bar{i}, \bar{j}) \in L\left(a, a_{1}, a_{2}\right)$

$$
\mathbf{P}\{B(\bar{i}) \cap B(\bar{j})\}< \begin{cases}\Delta^{a_{1}} \delta, & a_{1}<a=a_{1}+k, \\ \Delta^{a}, & a_{1}<a<a_{1}+k, a \neq a_{2}+k, \text { или } a \leqslant a_{1}<k .\end{cases}
$$

Так как можно полагать $a_{1} \leqslant a_{2}$, осталось рассмотреть случай $a \leqslant a_{1}=a_{2}=k$. Если $a<k$, то в силу леммы 1

$$
\mathbf{P}\{B(\bar{i}) \cap B(\bar{j})\}<\Delta^{k-1} \leqslant \Delta^{a} .
$$

Если $a=a_{1}=a_{2}=k$ и существует связная компонента множества $U(\bar{i}, \bar{j})$, не содержащая ни одной из точек $i_{1}, \ldots, i_{k}$ (или $\left.j_{1}, \ldots, j_{k}\right)$ ), то в силу леммы 1

$$
\mathbf{P}\{(\bar{i}, \bar{j})\}<\Delta \delta \leqslant \Delta^{k} .
$$

Теперь рассмотрим случай, когда $a=a_{1}=a_{2}=k$ и в каждой компоненте $U(\bar{i}, \bar{j})$ присутствуют точки $i_{s}$ и $j_{q}$. Не ограничивая общности, можно считать, что $s=q$. По условию леммы 2 случай, когда $i_{s}-j_{s}=i_{q}-j_{q}, s, q=1, \ldots, k$, невозможен, так как в этом случае $(\bar{i}, \bar{j}) \notin I_{n s}$. Тогда, не ограничивая общности, можно считать, что $i_{1}-j_{1} \neq i_{2}-j_{2}$. По формуле полной вероятности

$$
\begin{aligned}
\mathbf{P}\{B(\bar{i}) \cap B(\bar{j})\}= & \mathbf{P}\left\{\eta\left(i_{1}, i_{2}\right)=1, \eta\left(j_{1}, j_{2}\right)=1\right\} \\
& \times \mathbf{P}\left\{B(\bar{i}) \cap B(\bar{j}) / \eta\left(i_{1}, i_{2}\right)=1, \eta\left(j_{1}, j_{2}\right)=1\right\} \\
& +\mathbf{P}\left\{\eta\left(i_{1}, i_{2}\right) \eta\left(j_{1}, j_{2}\right)=0, B(\bar{i}) \cap B(\bar{j})\right\} .
\end{aligned}
$$

Второе слагаемое, очевидно, равно нулю. Второй сомножитель, очевидно, не превосходит $\Delta^{k-2}$. Покажем, что первый сомножитель не превосходит $\Delta^{2}$. Будем придерживаться схемы доказательства леммы 3 работы [1].

Необходимым и достаточным условием выполнения события

$$
\left\{\eta\left(i_{1}, i_{2}\right)=1, \eta\left(j_{1}, j_{2}\right)=1\right\}
$$

является выполнение равенств

$$
X_{i_{1}+t}=g\left(X_{i_{2}+t}\right), X_{j_{1}+1}=h\left(X_{j_{2}+t}\right), \quad g, h \in H, \quad t=0, \ldots, n-1 .
$$

Обозначим через $w$ число различных неизвестных, участвующих в системе (9). Если число свободных неизвестных в (9) равно $w^{\prime}$, то остальные однозначно определяются ими и подстановками $g$ и $h$, которых существует не более $|H|^{2}$. Поэтому справедливо неравенство

$$
\mathbf{P}\left\{\eta\left(i_{1}, i_{2}\right)=1, \eta\left(j_{1}, j_{2}\right)=1\right\} \leqslant v^{w^{\prime}-w}|H|^{2} .
$$

Доказательство, что свободные переменные можно выбрать так, что $w^{\prime}-w \geqslant 2 n$, совпадает с доказательством леммы 3 работы [1] (случай $r=2, a=2$ ). Таким образом,

$$
\mathbf{P}\left\{\eta\left(i_{1}, i_{2}\right)=1, \eta\left(j_{1}, j_{2}\right)=1\right\} \leqslant \Delta^{2} .
$$

Справедлива оценка

$$
\mathbf{P}\{B(\bar{i}) \cap B(\bar{j})\} \leqslant \Delta^{k}
$$


Поэтому при $(\bar{i}, \bar{j}) \in L\left(a, a_{1}, a_{2}\right), a_{1} \leqslant a_{2}$,

$$
\mathbf{P}\{B(\bar{i}) \cap B(\bar{j})\}< \begin{cases}\delta \Delta^{a_{1}}, & a_{1}<a=a_{1}+k, \\ \Delta^{a} & \text { в остальных случаях. }\end{cases}
$$

В первом случае очевидно, $a_{2}=k$. В силу неравенства (10) и определения множеств $L\left(a, a_{1}, a_{2}\right)$

$$
\begin{aligned}
\sum_{L\left(a_{1}+k, a_{1}, k\right)} \mathbf{P}\{B(\bar{i}) \cap B(\bar{j})\} & \leqslant \sum_{J\left(a_{1}\right)} \Delta^{a_{1}} \sum_{J} \delta=\lambda_{1}\left|J\left(a_{1}\right)\right| \Delta^{a_{1}} \\
& \leqslant \lambda_{1}\left(\begin{array}{c}
N \\
a_{1}
\end{array}\right)\left(\begin{array}{c}
k-1 \\
a_{1}-1
\end{array}\right) n^{k-a_{1}} \Delta^{a_{1}} .
\end{aligned}
$$

Пусть

$$
L(a)=\bigcup_{a_{1}=1}^{k} \bigcup_{a_{2}=1}^{k} L\left(a, a_{1}, a_{2}\right)
$$

В [8] показано, что

$$
|L(a)| \leqslant \frac{(2 k) !}{(k !)^{2}}\left(\begin{array}{c}
N \\
a
\end{array}\right)\left(\begin{array}{c}
2 k-1 \\
a-1
\end{array}\right) n^{2 k-1} \leqslant\left(\begin{array}{c}
N \\
a
\end{array}\right)(4 n)^{2 k-1},
$$

где последнее неравенство опирается на легко проверяемую оценку

$$
\frac{(2 k) !}{(k !)^{2}} \leqslant 2^{2 k-1}
$$

Отсюда находим, что

$$
\begin{aligned}
\sum_{(\bar{i}, \bar{j}) \in I_{n, s}} \mathbf{E}(\eta(\bar{j}) \eta(\bar{i})) & \leqslant \lambda_{1} \sum_{a=1}^{k-1}\left(\begin{array}{l}
N \\
a
\end{array}\right)\left(\begin{array}{c}
k-1 \\
a-1
\end{array}\right) n^{k-a} \Delta^{a}+\sum_{a=1}^{2 k-1}\left(\begin{array}{c}
N \\
a
\end{array}\right)(4 n)^{2 k-1} \Delta^{a} \\
& \leqslant \lambda_{1} \psi+(4 n)^{2 k-1} \sum_{a=1}^{\infty} \frac{(N \Delta)^{a+1}}{(a+1) !}
\end{aligned}
$$

Лемма 3. $E с л и ~ n / v \geqslant \ln v+1$, то

$$
\sum_{(\bar{i}, \bar{j}) \in I_{*}} \mathbf{E}(\eta(\bar{j}), \eta(\bar{i})) \leqslant \frac{12 \lambda_{2}}{v} .
$$

Доказательство. Не ограничивая общности можно считать, что $i_{1}<j_{1}, j_{1}-i_{1}=d$, графически этот случай выглядит следующим образом:

$$
\begin{aligned}
& X_{i_{1}} \overbrace{\substack{\downarrow h_{1} \\
X_{j_{1}}}}^{n} \overbrace{\substack{\downarrow h_{1}, g_{1} \\
X_{i_{1}+n-1}}}^{n-d} \overbrace{\substack{\downarrow g_{1} \\
X_{i_{2}}}}^{n-d} X_{j_{1}+n-1}
\end{aligned}
$$

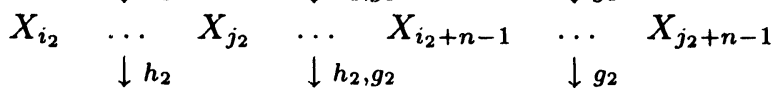

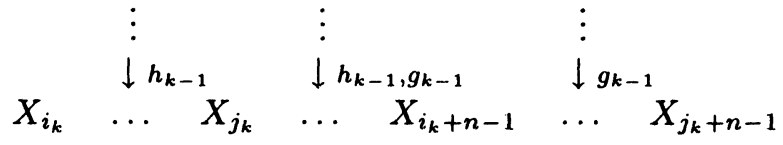


На диаграмме стрелками показана подстановочная связь знаков отрезков. Например, знаки отрезков

$$
Y_{j_{1}}(n-d)=\left(X_{j_{1}}, \ldots, X_{j_{1}+n-1}\right), \quad Y_{j_{2}}(n-d)=\left(X_{j_{2}}, \ldots, Y_{j_{2}+n-1}\right)
$$

связаны подстановками $h_{1}$ и $g_{1}$, то есть

$$
X_{j_{2}+t}=h_{1}\left(X_{j_{1}+t}\right), \quad X_{j_{2}+t}=g_{1}\left(X_{j_{1}+t}\right), \quad t=0, \ldots, n-1 .
$$

Обозначим через $P((\bar{i}, \bar{j}), d),(\bar{i}, \bar{j}) \in I_{s}$, вероятность

$$
\mathbf{P}(B(\bar{i}) \cap B(\bar{j}))
$$

при $i_{1}-j_{1}=\ldots=i_{k}-j_{k}=d$ и оценим ее.

Пусть $Y_{j_{1}}(n-d)$ содержит $m$ различных элементов $a_{1}, a_{2}, \ldots, a_{m}$ множества $A$. Таких $n-d$ выборок ровно $\sigma(n-d, m)$. Далее, пусть $Y_{i_{1}}(d)$ и $Y_{i_{1}+n-1}(d)$ содержат $m_{1}$ и $m_{2}$ различных элементов, соответственно, причем ровно $s_{1}$ и $s_{2}$ из них совпадают (без учета кратности) с элементами выборки $Y_{j_{1}}(n-d)$. Пусть остальные $m_{1}-s_{1}$ и $m_{2}-s_{2}$ элементов - это $b_{1}, \ldots, b_{m_{1}-s_{1}}$ и $c_{1}, \ldots, c_{m_{2}-s_{2}}$. Обозначим через $r$ минимум чисел $m_{1}-s_{1}$ и $m_{2}-s_{2}$. Тогда нетрудно убедиться в справедливости формулы

$$
\begin{aligned}
P\{(\bar{i}, \bar{j}), d\} \leqslant & \frac{1}{v^{k(n+d)}} \sum_{m=1}^{\min (n-d, v)} \sigma(n-d, m) \\
& \times \sum_{\left(a_{1}, \ldots, a_{m}\right) \in \nu_{m}(A)} \sum_{m_{1}=1}^{\min (d, v)} \sigma\left(d, m_{1}\right) \sum_{m_{2}=1}^{\min (d, v)} \sigma\left(d, m_{2}\right) \\
& \times \sum_{s_{1}=0}^{\min \left(m, m_{1}\right)}(m)_{s_{1}}\left(\begin{array}{c}
m_{1} \\
s_{1}
\end{array}\right) \sum_{s_{2}=0}^{\min \left(m, m_{2}\right)}(m)_{s_{2}}\left(\begin{array}{c}
m_{2} \\
s_{2}
\end{array}\right) \\
& \times \sum_{\left(b_{1}, \ldots, b_{m_{1}-s_{1}}\right) \in \nu_{m_{1}-s_{1}}\left(A \backslash\left(a_{1}, \ldots, a_{m}\right)\right)}|H|^{k-1}\left((v-m)_{r}\right)^{k-1} . \\
& \times \sum_{\left(c_{1}, \ldots, c_{m_{2}-s_{2}}\right) \in \nu_{m_{2}-s_{2}}\left(A \backslash\left(a_{1}, \ldots, a_{m}\right)\right)}
\end{aligned}
$$

Рассмотрим сначала случай $d=1$, в этом случае

$$
\begin{aligned}
\Sigma_{1}= & P\{(\bar{i}, \bar{j}), 1\} \leqslant\left(\begin{array}{l}
N \\
k
\end{array}\right) \frac{|H|^{k-1}}{v^{k(n+1)}} \sum_{m=1}^{v} \sigma(n-1, m)(v)_{m} \\
& \times \sum_{s_{1}=0}^{1} \sum_{s_{2}=0}^{1}(m)_{s_{1}}(m)_{s_{2}}(v-m)_{1-s_{1}}(v-m)_{1-s_{2}}(v-m)_{1-\max \left(s_{1}, s_{2}\right)}^{k-1}=\sum_{m=1}^{v} \Sigma_{1, m}
\end{aligned}
$$

где при $m=1, \ldots, m$

$$
\Sigma_{1, m}=\lambda_{2} \frac{1}{v^{k} v^{n}} \sigma(n-1, m)(v)_{m}\left(m^{2}+2 m(v-m)+(v-m)^{k+1}\right) .
$$

Заметим, что

$$
\Sigma_{1, v} \leqslant \lambda_{2} \frac{1}{v^{k} v^{n}} \frac{v^{n-1}}{v !}(v !) v^{2} \leqslant \lambda_{2} \frac{1}{v^{k-1}}
$$


Далее,

$$
\begin{aligned}
\Sigma_{1}^{1} & =\frac{\lambda_{2}}{v^{k} v^{n}} \sum_{m=1}^{v-1} \sigma(n-1, m)\left(v_{m}\right) 2 m v \\
& \leqslant \frac{\lambda_{2}}{v^{k} v^{n}} \sum_{m=1}^{v-1} \frac{m^{n-1}}{m !}(v)_{m} 2 m v \leqslant \frac{2 \lambda_{2}}{v^{k-2}} \frac{v !}{v^{n}} \sum_{m=1}^{v-1} \frac{m^{n-1}}{m !}, \\
\Sigma_{1}^{2} & =\frac{\lambda_{2}}{v^{k} v^{n}} \sum_{m=1}^{v-1} \sigma(n-1, m)(v)_{m}(v-m)^{k+1} \leqslant \lambda_{2} \frac{v !}{v^{n}} \sum_{m=1}^{v-1} \frac{m^{n-1}}{m !} .
\end{aligned}
$$

В лемме 5 работы [1] показано, что если $n / v \geqslant \ln v+1$, то для всех $m=1, \ldots, v-1$ справедливо неравенство

$$
\frac{m^{n-1}}{m !} \leqslant \exp \{(1 / v-1)(v-m-1)\} \frac{(v-1)^{n-1}}{(v-1) !}
$$

Поэтому, учитывая, что $1-1 / v<\exp \{-1 / v\}$, получаем, что

$$
\begin{aligned}
\frac{v !}{v^{n}} \sum_{m=1}^{v-1} \frac{m^{n-1}}{m !} & \leqslant \frac{v !}{v^{n}} \sum_{m=1}^{v-1} \exp \{(1 / v-1)(v-m-1)\} \frac{(v-1)^{n-1}}{(v-1) !} \\
& \leqslant(1-1 / v)^{n-1} \sum_{m=1}^{v-1} \exp \{(1 / v-1)(v-m-1)\} \\
& \leqslant(1-1 / v)^{v \ln v+v-1} \frac{1}{1-e^{1 / v-1}} \\
& \leqslant 3 \exp \{-(1 / v) v \ln v\}=3 / v
\end{aligned}
$$

Таким образом,

$$
\Sigma_{1} \leqslant \lambda_{2} \frac{1}{v^{k-1}}+\lambda_{2} \frac{2}{v^{k-2}} \frac{3}{v}+\lambda_{2} \frac{3}{v} \leqslant 10 \lambda_{2} \frac{1}{v} .
$$

Кроме того, для любого $2 \leqslant d \leqslant n-1$

$$
\begin{aligned}
P((\bar{i}, \bar{j}), d) & \leqslant \Delta^{k-1} \frac{1}{v^{n} v^{k d}} \sum_{m=1}^{\min (n-d, v)} \sigma(n-d, m)(v)_{m}(v-m) !(v-m)^{d(k-2)} v^{2 d} \\
& \leqslant \Delta^{k-1} \frac{v !}{v^{n}} \sum_{m=1}^{\min (n-d, v)} \sigma(n-d, m) .
\end{aligned}
$$

Далее,

$$
\begin{aligned}
\sum_{(\bar{i}, \bar{j}) \in I_{s}} \mathrm{E} \eta(\bar{j}) \eta(\bar{i}) & =\Sigma_{1}+\left(\begin{array}{c}
N \\
k
\end{array}\right) \sum_{d=2}^{n-1} P((\bar{i}, \bar{j}), d) \\
& =\frac{10 \lambda_{2}}{v}+\lambda_{2} \frac{v !}{v^{n}} \sum_{d=2}^{n-1} \sum_{m=1}^{\min (n-d, v)} \sigma(n-d, m)
\end{aligned}
$$

Для оценки последнего слагаемого воспользуемся рассуждениями, аналогичными доказательству леммы 4 в [1]. 
Пусть $2 \leqslant d \leqslant n-\ln v$. Тогда $v \ln v \leqslant n-d \leqslant n-2$. Учитывая неравенства

$$
n / v \geqslant \ln v+1, \quad(1-1 / v)<\exp \{-1 / v\}
$$

получим, что

$$
\begin{aligned}
\frac{m^{n-d}}{m !} \frac{(m+1) !}{(m+1)^{n-d}} & \leqslant(m+1)\left(1-\frac{1}{m+1}^{n-d}\right) \\
& \leqslant v\left(1-\frac{1}{v}\right)^{n-d}<v \exp \{-(1 / v) v \ln v\}=1
\end{aligned}
$$

Откуда,

$$
\begin{aligned}
\frac{v !}{v^{n}} \sum_{d=2}^{n-1} \sum_{m=1}^{\min (n-d, v)} \sigma(n-d, m) & <\frac{v !}{v^{n}} \sum_{d=2}^{n-1} \sum_{m=1}^{\min (n-d, v)} \frac{v^{n-d}}{v !} \\
& \leqslant \sum_{d=2}^{n-1} \frac{1}{v^{d-1}} \leqslant \frac{1}{v-1} \leqslant \frac{2}{v}
\end{aligned}
$$

Продолжим доказательство теоремы 1 . В силу лемм 2 и 3

$$
\begin{aligned}
\Lambda & \leqslant \psi \Delta+\lambda_{1} \delta+2 \psi^{2}+\lambda_{1}\left(2 \psi+\left((2 n)^{k}-1\right)(N \Delta)^{k-1}\right)+\lambda_{1} \psi+\Psi^{\prime}+12 \lambda_{2} \frac{1}{v} \\
& \leqslant\left(\psi \Delta+2 \psi^{2}+\Psi^{\prime}\right)+\lambda_{1}\left(\delta+3 \psi+\left((2 n)^{k}-1\right)(N \Delta)^{k-1}\right)+\frac{12 \lambda_{2}}{v} .
\end{aligned}
$$

Замечая, что $\delta<\Delta^{k-1}$ и $\psi \Delta+2 \psi+\Psi^{\prime} \leqslant \Psi$, находим, что

$$
\Lambda \leqslant \Psi+\lambda_{1}\left(3 \psi+(2 n)^{k}(N \Delta)^{k-1}\right)+\frac{12 \lambda_{2}}{v} .
$$

Далее, по свойствам расстояния по вариации

$$
\begin{aligned}
d\left(F_{\xi_{k}}, \Pi_{\lambda_{1}}\right) & \leqslant d\left(F_{\xi_{k}}, \Pi_{\lambda}\right)+d\left(\Pi_{\lambda_{1}}, \Pi_{\lambda}\right) \leqslant \frac{1-e^{-\lambda}}{\lambda} \Lambda+\left|\lambda_{1}-\lambda\right| \\
& \leqslant\left(\frac{1-e^{-\lambda_{1}}}{\lambda_{1}}+\left|\frac{1-e^{-\lambda_{1}}}{\lambda_{1}}-\frac{1-e^{-\lambda}}{\lambda}\right|\right) \Lambda+\psi .
\end{aligned}
$$

Заметим, что функция $f(x)=\left(1-e^{-x}\right) / x$ дифференцируема на любом отрезке $\left[x_{1}, x_{2}\right], 0<x_{1}, x_{2}<\infty$, и

$$
f^{\prime}(x)=-\frac{1-e^{-x}-x e^{-x}}{x^{2}}
$$

Применяя на отрезке $\left[\min \left(\lambda_{1}, \lambda\right), \max \left(\lambda_{1}, \lambda\right)\right]$ к $f(x)$ теорему Лагранжа о промежуточном значении, получаем, что

$$
\left|\frac{1-e^{-\lambda_{1}}}{\lambda_{1}}-\frac{1-e^{-\lambda}}{\lambda}\right|=\left|\lambda_{1}-\lambda\right|\left|\frac{1-e^{-\theta}-\theta e^{-\theta}}{\theta^{2}}\right| \leqslant \psi\left|\frac{1-e^{-\theta}-\theta e^{-\theta}}{\theta^{2}}\right|
$$

при некотором $\theta \in\left[\min \left(\lambda_{1}, \lambda\right), \max \left(\lambda_{1}, \lambda\right)\right]$. Заметим, что при любом $0<x<\infty$ справедливы оценки $\left|f^{\prime}(x)\right|<1$ и $\left|f^{\prime}(x)\right|<1 / x^{2}$ и, значит, $\left|f^{\prime}(\theta)\right|<1$ и $\left|f^{\prime}(\theta)\right|<1 / \theta^{2}$. 
Кроме того, функция $\left|f^{\prime}(x)\right|$ при $x \geqslant 1$ убывает и $1 / x^{2}>1$ на интервале $(0,1)$. Тогда в силу неравенства

$$
\lambda \geqslant \min \left\{\lambda_{1},\left|\lambda_{1}-\psi\right|\right\}
$$

справедливы оценки

$$
\begin{aligned}
\left|f^{\prime}(v)\right| & <\min \left\{1,1 / v^{2}\right\} \leqslant \min \left\{1,\left(\min \left(\lambda_{1}, \lambda\right)\right)^{-2}\right\} \\
& \leqslant \min \left\{1,\left(\min \left\{\lambda_{1},\left|\lambda_{1}-\psi\right|\right\}\right)^{2}\right\} .
\end{aligned}
$$

Теорема 1 доказана.

Дохазательство следствия 1. Первое утверждение следствия нетрудно получить непосредственно из теоремы 1. Докажем второе утверждение.

Согласно условию, найдется константа $c$ и натуральное число $N_{0}$ такие, что для всех $N$ больших $N_{0}$

$$
\begin{aligned}
0<c<\lambda_{2} & =\left(\begin{array}{c}
N \\
k
\end{array}\right) \Delta^{k-1} \leqslant \frac{N^{k}}{k !} \Delta^{k-1} \leqslant \frac{N^{k}}{(k / 3)^{k}} v^{(v-n)(k-1)} \\
& \leqslant\left(\frac{3 N}{k}\right)^{k} v^{-v \ln v \cdot(k-1)} \leqslant\left(\frac{3 N}{k}\right)^{k} v^{-\ln v \cdot k} \leqslant\left(\frac{3 N}{k v^{\ln v}}\right)^{k} .
\end{aligned}
$$

Значит $k=o(N)$ и

$$
N \geqslant\left(\lambda_{2}(1 / \Delta)^{k-1}\right)^{1 / k}
$$

Поэтому для достаточно больших $N$

$$
\begin{aligned}
\lambda_{2} & =\left(\begin{array}{c}
N \\
k
\end{array}\right) \Delta^{k-1} \geqslant \frac{N^{k}}{k !} \Delta^{k-1} \frac{(N-1)_{k-1}}{N^{k-1}} \\
& \geqslant N \frac{N^{k-1}}{k^{k-1}} \Delta^{k-1}\left(1-\frac{k}{N}\right)^{k-1} \geqslant\left(\frac{N \Delta}{k}(1-k / N)(1 / \Delta)^{1 / k}\right)^{k-1} \lambda_{2}^{1 / k},
\end{aligned}
$$

и

$$
\begin{aligned}
\lambda_{2} & \geqslant \frac{N}{k}(1-k / N) \Delta^{(k-1) / k}=\frac{N}{k} \Delta^{(k-1) / k}(1+o(1)), \\
N & \geqslant \lambda_{2} k \Delta^{(k-1) / k}
\end{aligned}
$$

Поэтому

$$
\begin{aligned}
\varphi & =(4 n)^{2 k-1} N \Delta \leqslant(4 n)^{2 k-1} \Delta \lambda_{2} k \Delta^{(1-k) / k} \\
& \leqslant \lambda_{2} k(4 n)^{(2 k-1)} \Delta^{1 / k}
\end{aligned}
$$

Доказательство теоремы 2. Первые два утверждения теоремы вытекают из следствия 1. Докажем третье утверждение.

Если $k=o(\sqrt{n \ln v / \ln n})$, то с учетом того, что $\lambda_{2} / v \rightarrow 0$, получаем оценки

$$
\begin{aligned}
\ln \left(\lambda_{2} k(4 n)^{2 k-1} \Delta^{1 / k}\right) & \leqslant \ln \left(v k(4 n)^{2 k-1} \Delta^{1 / k}\right) \\
& \leqslant \ln v+\ln k+(2 k-1) \ln (4 n)-(n-v) / k \ln v \\
& \leqslant(k \ln v \ln k+k(2 k-1) \ln (4 n)-(n-v-k) \ln v) / k,
\end{aligned}
$$

и правая часть стремится к минус бесконечности. 
Теоремы 1 и 2 позволяют описать предельное распределение с.в.

$$
\tau_{k}=\tau_{k}(n, H)=\min \left\{N: \xi_{k}(n, N)>0\right\},
$$

равной длине последовательности $X$ до появления первого набора из $k H$-эквивалентных отрезков длины $n$, и с.в.

$$
\mu_{k}=\mu_{k}(N, H)=\max \left\{n: \xi_{k}>0\right\}
$$

равной максимальной длине наборов из $k H$-эквивалентных отрезков в последовательности $X$. Приведем соответствующие результаты без доказательства.

Следствие 2. Пустъ $N, n, v \rightarrow \infty u n / v-\ln v \rightarrow \infty$. Тогда прu $x>c>0$, если $k \geqslant 2$ фиксировано и $x=o\left(v^{1 / k}\right)$, то

$$
\mathbf{P}\left\{\tau_{k} \Delta^{(k-1) / k} \leqslant x\right\}=1-\exp \left\{-x^{k} / k !\right\}(1+o(1)),
$$

eсли $k \rightarrow \infty, k=o(\sqrt{n \ln v / \ln n}), x=o(\ln v) u x=o(k)$, mo

$$
\mathbf{P}\left\{\tau_{k} e \Delta^{(k-1) / k}-k-\ln \sqrt{2 \pi k} \leqslant x\right\}=\left(1-\exp \left\{-e^{-x}\right\}\right)(1+o(1)) .
$$

Следствие 3. Пусть $N, v \rightarrow \infty$,

$$
\left(\begin{array}{c}
N \\
k
\end{array}\right)|H|^{k-1} \geqslant v^{(k-1) v(\omega(v)+\ln (v))}
$$

где $\omega(v) \rightarrow \infty$ и въполнено условие $k=o(\sqrt{v \ln v})$. Тогда, если

$$
\left|\frac{\ln \left(\left(\begin{array}{c}
N \\
k
\end{array}\right)|H|^{k-1}\right)}{\ln \left(v^{k-1}\right)}-\left\langle\frac{\ln \left(\left(\begin{array}{c}
N \\
k
\end{array}\right)|H|^{k-1}\right)}{\ln \left(v^{k-1}\right.}\right\rangle\right|(k-1) \ln (v) \rightarrow \infty,
$$

где $\langle x\rangle-$ ближайшее $к x$ челое, то

$$
\mathbf{P}\left(\mu\left(S_{v}, N\right)=\left[\frac{\ln \left(\left(\begin{array}{c}
N \\
k
\end{array}\right)|H|^{k-1}\right)}{\ln \left(v^{k-1}\right)}\right]\right) \rightarrow 1
$$

ecлu

$$
\left(\frac{\ln \left(\left(\begin{array}{c}
N \\
k
\end{array}\right)|H|^{k-1}\right)}{\ln \left(v^{k-1}\right)}-\left\langle\frac{\ln \left(\left(\begin{array}{c}
N \\
k
\end{array}\right)|H|^{k-1}\right)}{\ln \left(v^{k-1}\right)}\right\rangle\right)(k-1) \ln (v) \rightarrow \ln (\lambda),
$$

где $0<\lambda<\infty$, mo

$$
\begin{gathered}
\mathbf{P}\left(\mu_{k}=\left\langle\frac{\ln \left(\left(\begin{array}{l}
N \\
k
\end{array}\right)|H|^{k-1}\right)}{\ln \left(v^{k-1}\right)}\right\rangle-1\right) \rightarrow e^{-\lambda}, \\
\mathbf{P}\left(\mu_{k}=\left\langle\frac{\ln \left(\left(\begin{array}{c}
N \\
k
\end{array}\right)|H|^{k-1}\right)}{\ln \left(v^{k-1}\right)}\right\rangle\right) \rightarrow 1-e^{-\lambda} .
\end{gathered}
$$


Заметим, что в случае, когда мощность $v$ алфавита $A$ не стремится к бескснечности, теорема 1 не дает возможности описать предельное распределение случайной величины $\xi_{k}$. Это обусловлено тем, что при фиксированном $v$ в качестве предельного распределения для $\xi_{k}$ выступает сложное распределение Пуассона. Условия сходимости функции распределения $\xi_{k}$ к сложному распределению Пуассона описывается следующей теоремой.

Пусть

$$
\lambda_{3}=\lambda_{3}(H, n, N)=\left(1-1 / v^{k-1}\right)\left(\begin{array}{l}
N \\
k
\end{array}\right) \Delta^{k-1} .
$$

Теорема 3. Пусть $N, n \rightarrow \infty, v$ фиксировано, $k=o(\sqrt{n / \ln n})$ и существует предел $\lambda_{0}^{\prime}=\lim _{N \rightarrow \infty} \lambda_{3}, 0<\lambda_{0}^{\prime}<\infty$. Тогдa

$$
\mathbf{E} z^{\xi_{k}} \rightarrow \exp \left(\lambda_{0}^{\prime}(z-1) /\left(1-z / v^{k-1}\right)\right)
$$

при фиксированном $k u$

$$
\mathbf{E} z^{\xi_{k}} \rightarrow \exp \left(\lambda_{0}^{\prime}(z-1)\right)
$$

$n p u k \rightarrow \infty$.

Доказателъство. Будем использовать метод Стейна для доказательства сложной пуассоновской аппроксимации (см. [11]) и придерживаться в ряде случаев схем рассуждений, изложенных в [6] и других работах А. М. Зубкова и В. Г. Михайлова, посвященных повторениям и совпадениям $s$-цепочек.

Пусть $J=\left\{\bar{i}: \bar{i}=\left(i_{1}, \ldots, i_{k}\right), 1 \leqslant i_{1}<\ldots<i_{k} \leqslant N\right\}$ и для любого $\bar{i} \in J$ пусть $J^{\prime}(\bar{i})$ - множество таких наборов $\bar{j} \in J, \bar{j} \neq \bar{i}$, что хотя бы один из отрезков $\left[i_{1}, i_{1}+3 n-1\right], \ldots,\left[i_{k}, i_{k}+3 n-1\right]$ пересекается хотя бы с одним из отрезков $\left[j_{1}, j_{1}+3 n-1\right], \ldots,\left[j_{k}, j_{k}+3 n-1\right]$. Пусть

$$
\begin{aligned}
U(\bar{i})= & \left\{\bar{j} \in J: \bar{j} \neq \bar{i},-n<i_{1}-j_{1}=\ldots=i_{k}-j_{k}<n,\right. \\
& \left.\left|i_{r}-i_{s}\right|>3 n-1, r, s=1, \ldots, k\right\}, \\
V(\bar{i})= & J^{\prime}(\bar{i}) \backslash U(\bar{i}), \\
\lambda(m)= & \frac{1}{m} \sum_{\bar{i} \in J} \mathbf{E}\left(\eta(\bar{i}) I\left(\eta(\bar{i})+\sum_{\bar{j} \in U(\bar{i})} \eta(\bar{j})=m\right)\right), \quad m=1, \ldots, 2 n-1 .
\end{aligned}
$$

Тогда согласно теореме в [11], если $m \lambda(m) \downarrow 0$ при $m \uparrow \infty$, то

$d\left(F_{\hat{\xi}_{k}}, C P\left(\Lambda^{\prime}\right)\right) \leqslant C\left(\Lambda^{\prime}\right)\left(\sum_{i \in J}\left(\mathbf{E} \eta(\bar{i})\left(\mathbf{E} \eta(\bar{i})+\sum_{\bar{j} \in J(\bar{i})} \mathbf{E} \eta(\bar{j})\right)\right)+\sum_{\bar{i} \in J} \sum_{\bar{j} \in V(\bar{i})} \mathbf{E} \eta(\bar{j}) \eta(\bar{i})\right)$,

где

$$
\Lambda^{\prime}=\sum_{m=1}^{2 n-1} \lambda(m) d(m)
$$

где $d(m)$ - мера, сосредоточенная в точке $m$,

$$
C\left(\Lambda^{\prime}\right)=\min \left\{1, \frac{1}{\lambda(1)-2 \lambda(2)}\left(\frac{1}{4(\lambda(1)-2 \lambda(2))}+(\ln 2(\lambda(1)-2 \lambda(2)))_{+}\right)\right\} .
$$


Далее будем предполагать, что $n / v \geqslant \ln (v)+1$. Сумма, стоячая в правой части равенства (11), оцениеается аналогично теореме 1 (несимметричный случай):

$$
d\left(F\left(\xi_{k}\right), C P\left(\Lambda^{\prime}\right)\right) \leqslant C\left(\Lambda^{\prime}\right)\left(\Psi^{\prime}+\lambda_{2}\left(3 \psi^{\prime}+(6 n)^{k}(N \Delta)^{k-1}\right)\right),
$$

где

$$
\begin{aligned}
\psi^{\prime} & =(6 n)^{k-1} N \Delta \exp (N \Delta / 6 n) \\
\Psi^{\prime} & =(12 n)^{2 k-1} N \Delta \exp (N \Delta)
\end{aligned}
$$

Найдем величины $\lambda(i)$. Рассмотрим событие

$$
V(q, m)=\left\{\left(X_{q}, \ldots, X_{q+m-1}\right) \text { содержит все символы алфавита } A\right\} .
$$

Из теоремы 1 работы [4] с использованием неравенства Маркова следует, что

$$
\mathbf{P}(V(q, m)) \geqslant 1-v \exp (-m / v) .
$$

Рассмотрим набор из $k$ непересекающихся векторов $\left(X_{i_{s}}, \ldots, X_{i_{s}+2 n-L-1}\right)$, $s=1, \ldots, k$ длины $2 n-L, 1 \leqslant L \leqslant n-1$ и оценим вероятность события

$$
\begin{aligned}
D(L)=\left\{\forall r, s=1, \ldots, k:\left(X_{i_{s}}, \ldots, X_{i_{s}+n-1}\right) H\left(X_{i_{r}}, \ldots, X_{i_{r}+n-1}\right)\right. \\
\text { и } \left.\left(X_{i_{s}+n-L}, \ldots, X_{i_{s}+2 n-L-1}\right) H\left(X_{i_{r}+n-L}, \ldots, X_{i_{r}+2 n-L-1}\right)\right\} .
\end{aligned}
$$

Заметим, что

$$
\mathbf{P}\left(D(L) \cap V\left(i_{s}+n-L, L\right)\right)=|H|^{k-1} / v^{(2 n-L)(k-1)} .
$$

Тогда из неравенства (12) следует, что

$$
\begin{aligned}
\left.|\mathbf{P}(D(L))-| H\right|^{k-1} / v^{(2 n-L)(k-1)} \mid & =\mathbf{P}\left(D(L) \backslash V\left(i_{s}+n-L, L\right)\right) \\
& \leqslant v \exp (-L / v)|H|^{2(k-1)} / v^{(2 n-L)(k-1)} .
\end{aligned}
$$

Кроме того, пусть $F(L)$ - событие, заключающееся в том, что найдутся натуральное $1 \leqslant j \leqslant n-L-1$ и пара $r, s \in 1, \ldots, k$ такие, что векторы

$$
\left(X_{i_{s}+j, \ldots, X_{i_{s}+j+n-1}}\right), \quad\left(X_{i_{r}+j, \ldots, X_{i_{r}+j+n-1}}\right)
$$

не $H$-эквивалентны. Тогда

$$
\begin{aligned}
D(L \cap F(L))= & (D(L) \cap F(L)) \\
& \backslash(V(n-L-\lfloor(n-L) / 2\rfloor+1,\lfloor(n-L) / 2\rfloor) \cap V(n+1,\lfloor(n-L) / 2\rfloor)) \\
= & (D(L) \cap F(L)) \backslash V(n-L+1, L)
\end{aligned}
$$

и

$$
\begin{aligned}
& \mathbf{P}(D(L) \cap F(L)) \leqslant v \exp (-L / v)|H|^{2(k-1)} / v^{(2 n-L)(k-1)} \\
& \mathbf{P}(D(L) \cap F(L)) \leqslant 2 v \exp (-\lfloor(n-L) / 2\rfloor / v)|H|^{2(k-1)} / v^{(2 n-L)(k-1)}
\end{aligned}
$$


Таким образом, если $1 \leqslant m \leqslant n-1$, то

$$
\begin{aligned}
\mid \lambda(m)-(1 & \left.-1 / v^{k-1}\right)^{2}\left(\begin{array}{c}
N \\
k
\end{array}\right)|H|^{k-1} / v^{(n+m-1)(k-1)} \mid \\
& =\left|\lambda(m)-\lambda_{3}\left(1-1 / v^{k-1}\right) / v^{(m-1)(k-1)}\right| \\
& \leqslant 2\left(\begin{array}{c}
N \\
k
\end{array}\right) n^{2} v \exp (-n / 3 v)|H|^{2(k-1)} / v^{(n+m-1)(k-1)} \\
& \leqslant 2 \lambda_{3} n^{2} v \exp (-n / 3 v)|H|^{(k-1)} / v^{(m-1)(k-1)} .
\end{aligned}
$$

Для $n \leqslant m \leqslant 2 n-2$

$$
\begin{aligned}
\mid \lambda(m)-\left(\lambda_{3} / m\right)(1 & \left.-1 / v^{k-1}\right)\left(2 n-m+2 / v^{k-1}\right) / v^{(m-1)(k-1)} \mid \\
& \leqslant \lambda_{3} n^{2} v \exp (-n / 3 v)\left(1-1 / v^{k-1}\right)^{-1}|H|^{k-1} / v^{(m-1)(k-1)}, \\
\left|\lambda(2 n-1)-\lambda_{3}\left(1-1 / v^{k-1}\right)^{-1}(2 n-1) / v^{(2 n-2)(k-1)}\right| & \leqslant \lambda_{3} v \exp (-n / 3 v)\left(1-1 / v^{k-1}\right)^{-1}\left(|H|^{k-1}+1\right) / v^{(2 n-2)(k-1)} .
\end{aligned}
$$

Рассмотрим сложное распределение Пуассона $C P(\Theta)$, где

$$
\Theta=\sum_{m=1}^{\infty} \theta(m) d(m), \quad \theta(m)=\lambda_{3}\left(1-1 / v^{k-1}\right) / v^{(m-1)(k-1)}
$$

Справедливы соотношения

$$
\begin{aligned}
d\left(F\left(\xi_{k}\right), C P(\Theta)\right) \leqslant & d\left(F\left(\xi_{k}\right), C P\left(\Lambda^{\prime}\right)\right)+d\left(C P\left(\Lambda^{\prime}\right), C P(\Theta)\right) \\
\leqslant & C\left(\Lambda^{\prime}\right)\left(\Psi^{\prime}+\lambda_{2}\left(3 \psi^{\prime}+(6 n)^{k}(N \Delta)^{k-1}\right)\right) \\
& \quad+\sum_{m=1}^{2 n-1}|\lambda(m)-\theta(m)|+\sum_{m=2 n}^{\infty} \theta(m) \\
\leqslant & \left(\Psi^{\prime}+\lambda_{2}\left(3 \psi^{\prime}+(6 n)^{k}(N \Delta)^{k-1}\right)\right) \\
& \quad+2 \lambda_{3} n^{2} \exp (-n / 3 v)|H|^{k-1} v\left(v^{k-1} /\left(v^{k-1}-1\right)\right)^{2} \\
\leqslant & \Psi^{\prime}+\lambda_{3}\left(6 \psi^{\prime}+2(6 n)^{k}(N \Delta)^{k-1}+16 v n^{2} \exp (-n / 3 v)|H|^{k-1}\right),
\end{aligned}
$$

где правая часть стремится к нулю.

Теорема доказана.

Заметим, что в ходе доказательства была получена явная оценка расстояния по вариации между распределением с.в. $\xi_{k}$ и сложным распределением Пуассона $C P(\Theta)$.

Автор благодарен В. Г. Михайлову за обсуждение результатов данной работы и ряд полезных замечаний.

4 Дискретная математика, т.14 №1 


\section{Список литературы}

1. Буравлев С. М., Повторения с точностью до перестановки в последовательности независимых испытаний. Дискретная математиха (1999) 11, №1, 53-75.

2. Буравлев С. М., Повторения с точностью до перестановок, образующих латинский прямоугольник. Дисхретная математиха (2000) 12, №1, 24-46.

3. Зубков А. М., Михайлов В. Г., Предельные распределения случайных величин, связанные с длинными повторениями в последовательности независимых испытаний. Теория вероятностей и ее применения (1974) 19, №1, 173-181.

4. Колчин В. Ф., Севастьянов Б. А., Чистяков В. П., Случайяые размещеяия. Наука, Москва, 1976.

5. Медведев Ю. И., Ивченко Г. И., Асимптотическое представление конечных разностей от степенной функции в произвольной точке. Теория вероятностей и ее применения (Г965) 10, №1, 45-61.

6. Михайлов В. Г., Предельные распределения случайных величин, связанных с многократными длинными повторениями в последовательности независимых испытаний. $\mathrm{Te}$ ория вероятностей и ее применения (1974) 19, №1, 182-187.

7. Михайлов В. Г., Предельная теорема Пуассона для числа $H$-совпадений цепочек. Обозрение прикладной и промышленяой математики (2000) 7, №1, 123-124.

8. Сачков В. Н., Введение в хомбинаторнъе методи дискретной математихи. Наука, Москва, 1982.

9. Феллер В., Введение в теорию вероятностей и ее приложения. т. 2, Мир, Москва, 1984.

10. Barbour A. D., Holst L., Janson S., Poisson approximation. Oxford Univ. Press, Oxford, 1992.

11. Roos M., Stein method for compound Poisson approximation: the local approach. Ann. Appl. Probab. (1994) 4, 1177-1187.

Статья поступила 02.07.2001. 